\title{
High mass Class I sources in M 17
}

\author{
M. Nielbock ${ }^{1,2}$, R. Chini $^{1}$, M. Jütte ${ }^{1}$, and E. Manthey ${ }^{1}$ \\ 1 Astronomisches Institut der Ruhr-Universität Bochum, Universitätsstr. 150/NA 7, 44780 Bochum, Germany \\ 2 ESO European Southern Observatory, Alonso de Cordova 3107, Casilla 19001, Santiago 19, Chile
}

Received 7 May 2001 / Accepted 16 July 2001

\begin{abstract}
The region of M 17 has been imaged at 10.5 and $20.0 \mu \mathrm{m}$ with the groundbased infrared camera MANIAC. In addition to a prominent diffuse emission bar $\left(4^{\prime} .5 \times 0^{\prime} .3\right)$ extending southeast to northwest at the interface between the $\mathrm{H}$ II region and the southwestern molecular cloud, the mosaic of 133 single frames at each wavelength revealed 22 compact sources. One of these sources is the Kleinmann-Wright-Object and another was previously identified as the ultra-compact $\mathrm{H}$ II region M 17-UC1. Combining the $N$-band and $Q$-band data with near infrared data yielded spectral energy distributions that classifies all sources to be of Class I. The observed luminosities were between 55 and $4775 L_{\odot}$, which suggests that these sources represent the youngest generation of massive early type stars in M 17 and are surrounded by relics of their protostellar clouds. The morphology of the 10.5 and $20.0 \mu \mathrm{m}$ emission towards some of the sources reveals flattened structures and may be the first evidence of the presence of circumstellar disks around massive stars.
\end{abstract}

Key words. ISM: dust, HII regions, individual objects: M 17 - stars: formation

\section{Introduction}

The energy output of the youngest stellar objects (YSOs) is dominated by reprocessed stellar light, originating in the dusty circumstellar environment of cocoons, shells or disks. Before the objects become visible at optical wavelengths, they manifest themselves as millimetre and/or infrared sources, depending on their evolutionary stage. In this framework, the amount of circumstellar material can be regarded as an age indicator, whereby decreasing thermal dust emission indicates increasing stellar age. Lada (1987) proposed to use the spectral energy distribution (SED), where the flux densites $F_{\lambda}$ of a source together with the corresponding wavelengths $\lambda$ are plotted. In order to measure thermal dust emission, he defined a spectral index $\alpha_{\mathrm{IR}}$, which describes the slope of this distribution between $2 \mu \mathrm{m}$ and 10 or $20 \mu \mathrm{m}$ according to:

$\alpha_{\mathrm{IR}}=\frac{\mathrm{d} \log \left(\lambda F_{\lambda}\right)}{\mathrm{d} \log (\lambda)}$

The earliest stage of evolution, in which most of the observed luminosity is derived from accretion, is characterised by values of $\alpha_{\mathrm{IR}}>0$. The corresponding objects are referred to as Class I. However, most YSOs are deeply embedded in the interior of dust clouds, and therefore their infrared SED is not solely a result of circumstellar

Send offprint requests to: M. Nielbock,

e-mail: mnielboc@eso.org emission but also influenced by heavy interstellar extinction. Chini \& Wargau (1998, hereafter CW) have pointed out that any ordinary star suffering from $A_{V}>80 \mathrm{mag}$, would appear as a Class I object.

The empirical classification scheme by Lada was based on observations of five low-mass sources from the Ophiuchus cloud. CW introduced another criterion based on observations of five high-mass stars, which may be used to determine the amount of circumstellar material in the absence of data longward of $4.8 \mu \mathrm{m}$. Their investigation of near-infrared (NIR) two-colour-diagrams (TCDs) showed that Class I sources - defined as such by their $\alpha_{\mathrm{IR}}-$ can also be characterised by the relations

$$
\begin{aligned}
& 0.26(J-K)+0.25<(K-L) \\
& 0.37(J-K)+0.80<(K-M)
\end{aligned}
$$

which are fairly independent of the amount of extinction and directly measure the emitting dust.

The Omega Nebula (M 17, NGC 6618) is a site of recent massive star formation (Chini et al. 1980, hereafter CEN). Its observed stellar content to date consisted of heavily reddened stars with spectral types between $\mathrm{O}$ and A. However, Manthey et al. (in prep.) recently found spectral types down to K 5 by means of $J H K$ photometry. The active process of star formation in this region is evident from a large number of embedded IR sources (CW), several cocoon stars (Chini 1982; Chini \& Krügel 1985, hereafter CK, CW) and an ultra-compact H II region (Felli et al. 1980). The present paper is the product of 


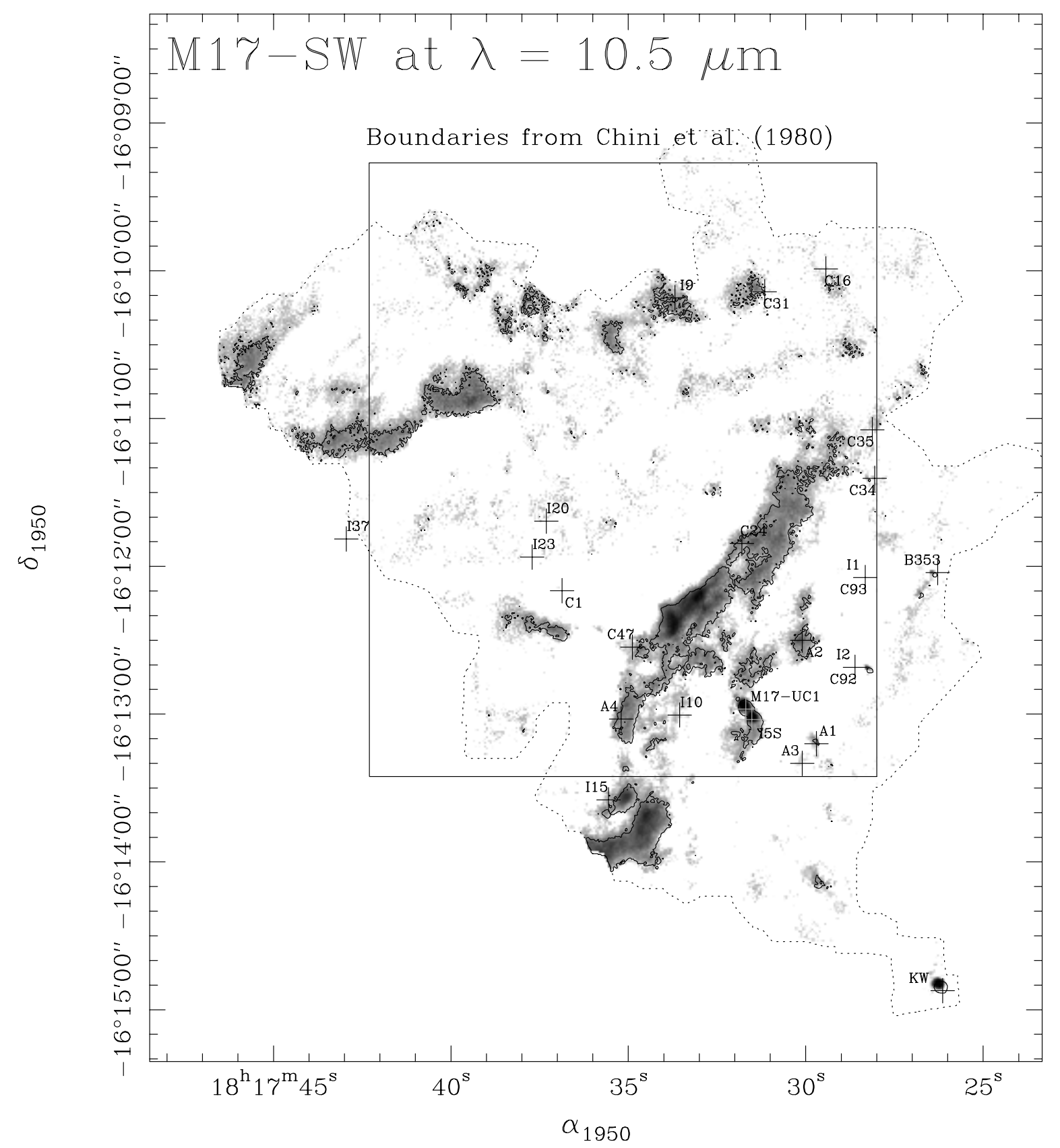

Fig. 1. Mid-infrared map of M 17 SW at $10.5 \mu \mathrm{m}$. The box indicates the area covered by the observations of CEN. The crosses indicate the location and the assumed positional uncertainty of $\pm 5^{\prime \prime}$ of the objects from which physical properties have been derived. They are labelled according to their naming in the literature (C: CEN, I: IRS, A: Anon, KW: Kleinmann-Wright-Object, M17-UC1: ultra-compact H II region). The contours indicate the $3 \sigma$ level of the map.

a longterm IR study of M 17 and reports on the detection of the first high-mass Class I objects, some of which may be surrounded by circumstellar disks.

\section{Observations and data reduction}

The observed region extends from $\alpha(1950)=18^{\mathrm{h}} 17^{\mathrm{m}} 20^{\mathrm{s}}$ to $18^{\mathrm{h}} 17^{\mathrm{m}} 55^{\mathrm{s}}$ and from $\delta(1950)=-16^{\circ} 08^{\prime} 00^{\prime \prime}$ to $-16^{\circ} 15^{\prime} 30^{\prime \prime}$ and thus covers the entire cluster field investigated by CEN at UBVRI and by CW at JHKLM.

\section{1. $1.25 \mu \mathrm{m}$ and $2.1 \mu \mathrm{m}$ imaging}

The $J$ and $K$ images were obtained at the $2.2 \mathrm{~m}$ telescope at Calar Alto in June 1998 with the infrared camera MAGIC. It is equipped with a Rockwell $256 \times 256 \mathrm{HgCdTe}$ detector, resulting in a pixel resolution of $1^{\prime \prime} 62 \times 1$ ". 62 and a field of view $(\mathrm{FOV})$ of $6.9 \times 6.9$. With a relative humidity of $\sim 80 \%$, the weather conditions were poor resulting in a seeing of about 2 . 5 . Standard chopping techniques were applied and the calibration is estimated to be accurate to about 0 . 05 both in $J$ and $K$. Further details of the 


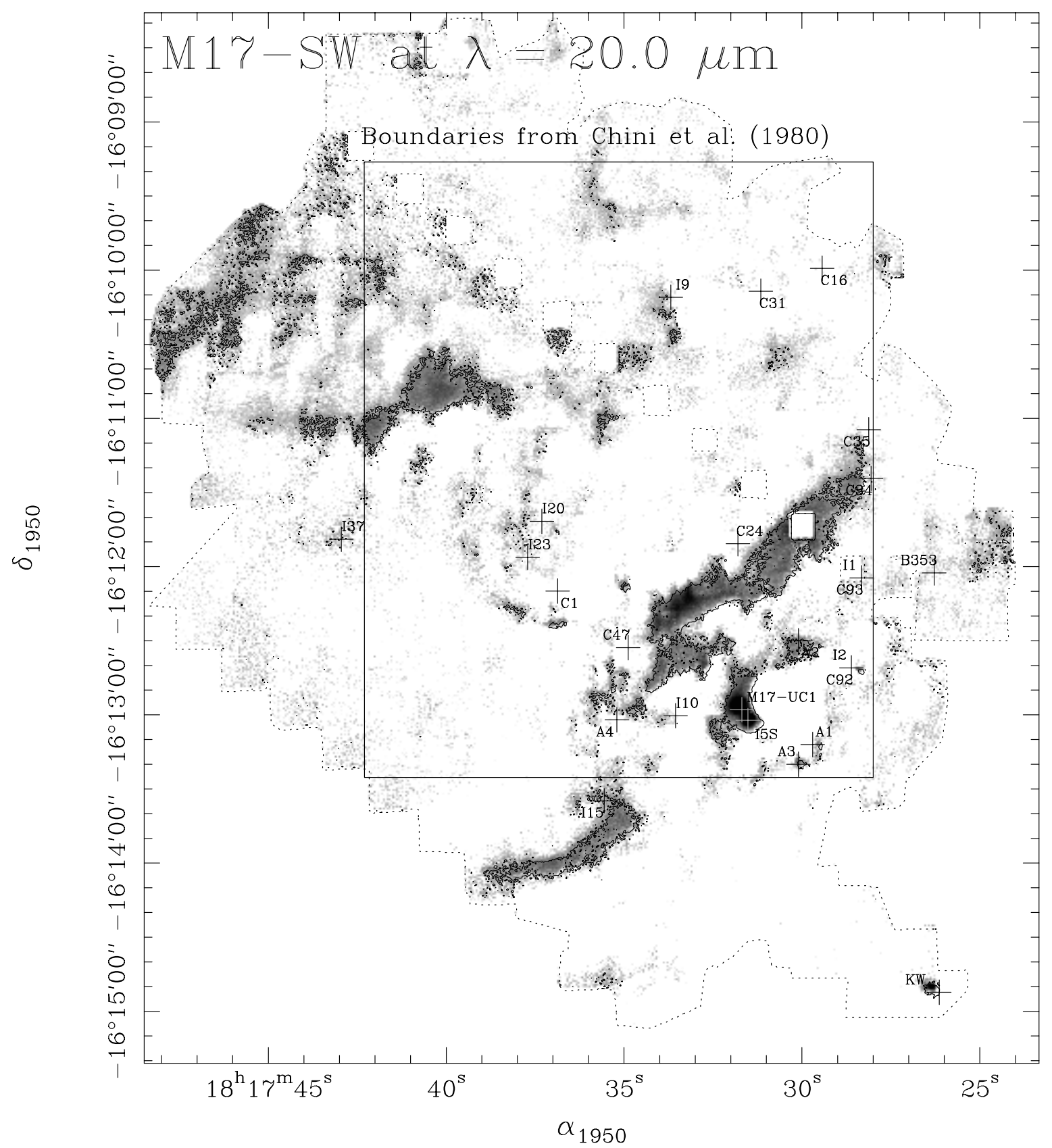

Fig. 2. Mid-infrared map of M $17 \mathrm{SW}$ at $20.0 \mu \mathrm{m}$. The diagonal series of holes in the map originates from a misalignment of the scans. For further explanation see text and Fig. 1.

observations and the data reduction are given elsewhere (Manthey et al., in prep.).

\section{2. $10.5 \mu \mathrm{m}$ and $20.0 \mu \mathrm{m}$ imaging}

The 10.5 and $20 \mu \mathrm{m}$ mosaics were obtained at the $2.2 \mathrm{~m}$ telescope on La Silla from June 28th to July 2nd 1997, using the infrared array camera MANIAC on its first scientific run. MANIAC was designed by Böker et al. (1997) to be a mapping instrument in the near- and mid-infrared that was to be upgraded in 5 phases. For this observations, the first phase was complete, providing a Rockwell $128 \times 128$ pixel Si:As detector for imaging purposes in
$N\left(\lambda_{\mathrm{c}}=10.50 \mu \mathrm{m}, \Delta \lambda=5.0 \mu \mathrm{m}\right)$ and $Q\left(\lambda_{\mathrm{c}}=20.00 \mu \mathrm{m}\right.$, $\Delta \lambda=5.40 \mu \mathrm{m})$ bands. This resulted in a pixel resolution of $0.337 \times 0^{\prime \prime} .337$ and a field of view of $43^{\prime \prime} .1 \times 43^{\prime \prime} .1$ for each frame.

A single frame was obtained by using conventional chopping techniques and an integration time of $30 \mathrm{~s}$. In order to minimise imbalances of the system, the chopping direction was set to $137^{\circ}$ with a throw of $26^{\prime \prime} \pm 0^{\prime \prime} 2$. To map the field of interest, we offset the telescope after each frame by the chop throw distance along the chopping direction. The length of an individual scan line was chosen in such a way that the signal decreased to zero at the start and the end of the scan. This procedure was necessary to 
obtain proper baselines for the reconstruction of the intensity profiles. Subsequent scan lines were offset by $45^{\prime \prime} 6$ in an east-west direction resulting in an overlap of 32.5 between adjacent frames. In total, we obtained 133 images at both wavebands. In addition, 18 particular sources of interest were re-observed with integration times of 120 to $180 \mathrm{~s}$ in order to achieve better $\mathrm{S} / \mathrm{N}$ ratios for those objects.

At the telescope, the relative pointing between the individual frames was achieved by offsetting each exposure from CEN 0 (SAO 161357). We adapted the programme package MOPSI (Zylka 1995) for reconstructing the scan lines and the mosaic. CEN 0, 2 and 3 (SAO 161359) were used for adjusting the coordinates resulting in a pointing accuracy of about $\pm 5^{\prime \prime}$ for a single frame. The flux calibration sources $\tau^{4}$ Eri, $\lambda \mathrm{Gru}, \eta \mathrm{Sgr}$ and $\lambda$ Sgr were taken from Gehrtz et al. (1974), Hanner et al. (1985), IRTF Photometry Manual (1990), Rieke et al. (1985) and Tokunaga (1984). The calibration uncertainties of $25 \%$ in $N$ and $10 \%$ in $Q$ are mainly due to the correction for the atmospheric extinction which suffered from highly variable conditions.

The ultra-compact $\mathrm{H}$ II region $\mathrm{M}$ 17-UC1 was reobserved with the mid-infrared camera TIMMI2 in $N$ at the ESO $3.6 \mathrm{~m}$ telescope on January 27th, 2001. Its detector is a $320 \times 240$ pixel Santa Barbara Si:As IBC array with a pixel resolution of $0 . ' 2$, resulting in a FOV of $64^{\prime \prime} \times 48^{\prime \prime}$. Chopping in declination and nodding in right ascension has been applied with a beam throw of $20^{\prime \prime}$, resulting in two positive and two negative signals for each source within one image. Atmospherical conditions appeared to be excellent during observing, achieving diffraction limited imaging with a seeing of $\sim 0.7^{\prime \prime}$. Images of $\theta$ Cen, $\alpha$ Tau and $\nu$ Vir were taken for calibration purposes, resulting in an overall calibration accuracy of $\sim 10 \%$. Because of the early stages of development in processing the TIMMI2 data, only the coadded raw images could be used for the photometry. The restoration of the 4 signals to one was achieved with MOPSI.

\section{Results}

In this section, we present the imaging data at 10.5 and $20 \mu \mathrm{m}$ and the photometric data of the compact sources contained in the MIR maps. The MIR data cover an area of several arcminutes in diameter, thus allowing a largescale, high-resolution investigation of the distribution of warm dust within M $17 \mathrm{SW}$.

\subsection{Diffuse dust emission}

Figures 1 and 2 show the final result of the observing/reduction procedure as described above and reveal an exciting view of M $17 \mathrm{SW}$ at 10.5 and $20.0 \mu \mathrm{m}$. The emission bar reaching 4.5 (2 pc) from NW to SE has been resolved into unprecedented detail revealing lots of embedded emission spots. The total flux determined from the maps is $4630 \mathrm{Jy}$ in $N$ and $16460 \mathrm{Jy}$ in $Q$. These values are consistent with the MIR luminosity in this wavelength regime of roughly $63800 L_{\odot}$ and a colour temperature of $205 \mathrm{~K}$. Summing up the luminosity of the compact sources within the same wavebands implies that their emission only accounts for about $6 \%$ of the total luminosity. Similar measurements for the emission bar give $1800 \mathrm{Jy}$ at $N$ and $4730 \mathrm{Jy}$ at $Q$, resulting in a MIR luminosity of about $15800 L_{\odot}$ and a colour temperature of $230 \mathrm{~K}$. Due to the small difference in temperature between the emission bar and the diffuse ambient medium, the strong surface brightness gradient must originate from density enhancements within the ridge.

A possible explanation for the origin of the ridge is the expanding ionisation front driven by CEN 1, and discussed previously by Felli \& Stanga (1987, hereafter FS). CEN 1 is an $\mathrm{O} 4 \mathrm{~V}$ star with a bolometric luminosity of $1.3 \times 10^{6} L_{\odot}$. It is located at $\alpha(1950)=18^{\mathrm{h}} 17^{\mathrm{m}} 36^{\mathrm{s}} .866$, $\delta(1950)=-16^{\circ} 12^{\prime} 09^{\prime \prime} .93$ and is the main exciting source within the dark cloud. The ionisation front is advancing to the SW and sweeping up the diffuse ISM at the edge of the molecular cloud and compressing it. This could be the cause of density waves that progress through the cloud triggering gravitational instabilities and consequently fragmentation and the formation of new stars. M 17 SW can therefore be regarded as a very impressive example of sequential star formation, as already discussed by Vallée \& Bastien (1996). In the light of this argument it is interesting to note that the majority of the investigated YSOs are located within this emission bar or to the southwest of it.

A second narrow emission ridge can be found in the MIR maps southeast of CEN 1. Interestingly, it seems to coincide with the southern edge of a feature which could be described as a dark rectangle and is clearly visible at NIR wavelengths. This rectangular structure is most likely caused by high interstellar extinction, originating from another dense molecular cloud or an isolated part of the whole cloud complex. The compact sources IRS 20 and IRS 23 are also located in this area, suggesting that this is another star forming region and that the ISM has been compressed by an ionisation front originating from CEN 1 which is moving along the line of sight.

\subsection{Compact sources}

The MIR-map positions of some of the visible and infrared stellar sources found by $\mathrm{CEN}$ and $\mathrm{CW}$ are marked with "C" (CEN) and "I" (IRS), respectively; four new sources are labelled with "A" (Anon). The Kleinmann-Wright source (Kleinmann \& Wright 1973), a deeply embedded Herbig Be star (Bunn et al. 1995), and M 17-UC1, an ultra compact H II region (Felli et al. 1980) are labelled as "KW" and "M17-UC1", respectively. The size of the crosses indicate the positioning uncertainty of $\pm 5^{\prime \prime}$. A total of 22 infrared sources within the field have been investigated in more detail. The ones labelled with CEN or IRS in Table 1 have been re-observed at $N$ and $Q$ with longer 
Table 1. Photometry of 22 infrared sources in M 17. The CEN and IRS numbers are taken from CEN and CW, respectively. Other designations originate from ${ }^{1}$ Kleinmann \& Wright (1973), ${ }^{2}$ Bunn et al. (1995), ${ }^{3}$ Hanson et al. (1997) and ${ }^{4}$ FS. The values in brackets are taken from Chini (1982), CK and CW. The flux density values for $N$ and $Q$ have been extracted from the MANIAC data within the quoted aperture radii.

\begin{tabular}{|c|c|c|c|c|c|c|c|c|c|}
\hline Source & $\begin{array}{c}\text { RA } \\
{[19:} \\
18^{\mathrm{h}} 17^{\mathrm{m}}\end{array}$ & $\begin{array}{c}\text { Dec } \\
50] \\
\mid-16^{\circ}\end{array}$ & & $J$ & $\begin{array}{l}\text { Flux density } \\
K^{[\mathbf{m J y}]}\end{array}$ & $N$ & $Q$ & $\begin{array}{c}\text { Aperture } \\
\text { radius } \\
N \text { and } Q\end{array}$ & $\begin{array}{c}\text { Other } \\
\text { designation }\end{array}$ \\
\hline $\mathrm{KW}^{1}$ & $\begin{array}{l}26.4 \\
26.6\end{array}$ & $\begin{array}{ll}14^{\prime} & 53^{\prime \prime} \\
12 & 01\end{array}$ & $\begin{aligned} 18 & \pm 3 \\
4 & \pm 1\end{aligned}$ & & $\begin{aligned} 639 & \pm 99 \\
13 & \pm 2\end{aligned}$ & $\begin{aligned} 86500 & \pm 8650 \\
1700 & \pm 150\end{aligned} \mid$ & $129300 \pm 13100$ & $\begin{array}{l}4^{\prime \prime} \\
2^{\prime \prime}\end{array}$ & $\begin{array}{c}\text { M17 SW-IRS1 } \\
{\text { B } 353^{3}}^{3}\end{array}$ \\
\hline CEN 34 & 28.1 & 1124 & $126 \pm 23$ & $(97 \pm 18)$ & $418 \pm 77(446 \pm 82)$ & $2500 \pm 730$ & $<6350$ & $3^{\prime \prime}$ & B358 ${ }^{3}$ \\
\hline CEN 35 & 28.1 & 1105 & $38 \pm 7$ & $(48 \pm 9)$ & $183 \pm 34(262 \pm 48)$ & $3100 \pm 310$ & $<6000$ & $3^{\prime \prime}$ & $\mathrm{B}_{333^{3}}$ \\
\hline IRS 1 & 28.3 & 1205 & $22 \pm 4$ & $(10 \pm 2)$ & $105 \pm 19(150 \pm 28)$ & $650 \pm 200$ & $<3250$ & $2^{\prime \prime}$ & B337 ${ }^{3}$, CEN 93 \\
\hline IRS 2 & 28.6 & 1241 & $46 \pm 8$ & $(41 \pm 7)$ & $167 \pm 31(200 \pm 37)$ & $8600 \pm 780$ & $30350 \pm 880$ & $3^{\prime \prime}$ & $\mathrm{B}_{331^{3}}, \mathrm{CEN} 92$ \\
\hline CEN 16 & 29.4 & 0959 & $219 \pm 40$ & $(178 \pm 33)$ & $183 \pm 34(183 \pm 34)$ & $6500 \pm 720$ & $<21500$ & $5^{\prime \prime}$ & B311 ${ }^{3}$ \\
\hline CEN 31 & 31.2 & 1009 & $126 \pm 23$ & $(114 \pm 21)$ & $139 \pm 26(146 \pm 27)$ & $25000 \pm 7590$ & $80600 \pm 13600$ & $10^{\prime \prime}$ & $\mathrm{B} 289^{3}$ \\
\hline CEN 24 & 31.8 & 1151 & $126 \pm 23$ & $(86 \pm 16)$ & $348 \pm 64(407 \pm 75)$ & $1900 \pm 320$ & $<7900$ & $3^{\prime \prime}$ & $\mathrm{B} 275^{3}$ \\
\hline CEN 47 & 34.9 & 1233 & $35 \pm 6$ & $(80 \pm 15)$ & $26 \pm 5 \quad(171 \pm 32)$ & $840 \pm 220$ & $12490 \pm 590$ & $2^{\prime \prime}$ & $\mathrm{B} 227^{3}$ \\
\hline IRS 5S & 31.5 & 1302 & $5 \pm 1$ & $(5 \pm 1)$ & $126 \pm 23(146 \pm 27)$ & $30700 \pm 2360$ & $250100 \pm 7830$ & $5^{\prime \prime}$ & $\mathrm{B} 284^{3}$ \\
\hline IRS $5 \mathrm{~N}$ & 31.7 & 1258 & $<2$ & & $20 \pm 4$ & $71200 \pm 6410$ & $259300 \pm 7520$ & $5^{\prime \prime}$ & ${\mathrm{M} 17-U C 1^{4}}^{4}$ \\
\hline IRS 9 & 33.1 & 1012 & & $(10 \pm 2)$ & $(56 \pm 10)$ & $24100 \pm 2170$ & $56400 \pm 2650$ & $7^{\prime \prime}$ & \\
\hline IRS 10 & 33.6 & 1300 & $6 \pm 1$ & $(5 \pm 1)$ & $(41 \pm 7)$ & $2200 \pm 450$ & & $2^{\prime \prime}$ & $\mathrm{B} 246^{3}$ \\
\hline IRS 15 & 35.6 & 1335 & $55 \pm 10$ & $(54 \pm 10)$ & $60 \pm 11 \quad(52 \pm 10)$ & $32700 \pm 2940$ & $91800 \pm 7700$ & $7^{\prime \prime}$ & $\mathrm{B} 215^{3}$ \\
\hline IRS 20 & 37.3 & 1142 & $<1$ & & $17 \pm 3 \quad(17 \pm 3)$ & $5400 \pm 480$ & $12200 \pm 2400$ & $5^{\prime \prime}$ & $\mathrm{B} 175^{3}$ \\
\hline IRS 23 & 37.7 & 1156 & $17 \pm 3$ & $(16 \pm 3)$ & $73 \pm 13(148 \pm 27)$ & $530 \pm 160$ & & $1^{\prime \prime} .5$ & \\
\hline IRS 37 & 42.9 & 1149 & $3 \pm 1$ & $(6 \pm 1)$ & $46 \pm 8 \quad(58 \pm 11)$ & $10800 \pm 2040$ & & $6^{\prime \prime}$ & $\mathrm{B} 91^{3}$ \\
\hline Anon 1 & 29.6 & 1312 & $6 \pm 1$ & & $15 \pm 3$ & $11400 \pm 1030$ & $8090 \pm 2600$ & $5^{\prime \prime}$ & \\
\hline Anon 2 & 30.3 & 1231 & $<2$ & & $<13$ & $12700 \pm 1140$ & $93650 \pm 30000$ & $10^{\prime \prime}$ & \\
\hline Anon 3 & 30.1 & 1320 & $<4$ & & $<13$ & $5540 \pm 525$ & $10040 \pm 3350$ & $7^{\prime \prime}$ & \\
\hline Anon 4 & 35.2 & 1302 & $<17$ & & & $9870 \pm 1260$ & & $5^{\prime \prime}$ & \\
\hline
\end{tabular}

integration times. The fluxes of the other sources have been determined from the maps. All of them are listed in Table 1 with their coordinates, JKNQ flux densities, the aperture radii used for $N Q$ photometry and further designations. The flux densities refer to an aperture size of typically $8^{\prime \prime}-12^{\prime \prime}$ in $J$ and $K$. The aperture sizes at $N$ and $Q$ had to be adjusted to the shape and extent of the different sources but were kept equal for each source. In order to verify any flux variations, former measurements at $J$ and $K$ from Chini (1982), CK and CW are quoted in brackets.

A comparison of our photometry with the values from the literature demonstrates excellent agreement in most cases. Only IRS 1, CEN 24, CEN 47, IRS 23 and IRS 37 show differences in the energy output that exceed the assumed photometrical errors. The changes reach values up to 0.9 in $J$, which is equivalent to an increase of a factor of 2.3 in flux density. The corresponding values for $K$ are $2 . \mathrm{m}^{\mathrm{m}} 0$ and a factor of 6.6 . Even though a misidentification of some of the sources cannot be totally ruled out, these inconsitencies suggest variability. If one takes also the sources into account that show changes in the mean value of the measured flux, which lie within the observational errors, a global trend becomes obvious: while the variation in $J$ is largely an increase, the $K$ flux seems to decrease. This may indicate that circumstellar matter evaporates or is blown away by the stellar wind and simultaneously the circumstellar extinction is diminished, exposing the hot surface of the star.

\section{The nature of the compact sources}

\subsection{The SEDs of individual sources}

Table 2 contains the physical properties of the sources. The spectral indices have been computed from the flux densities at $K$ and $N$. Where available, the spectral indices acoording to $K$ and $Q$ have also been added. The observed luminosity $L_{\mathrm{obs}}$ is the luminosity based on all data points found in the literature and plotted in the SEDs. For the morphology of the sources where a size larger than expected for diffraction limited source geometries has been detected, the projected linear dimensions at the major and minor axis are given. The spectral energy distributions in Fig. 3 allow us to derive temperatures. The spectral coverage of 14 sources was large enough to find evidence for mostly 2 , in some cases even 3 , temperature components. This was achieved by fitting Planck functions, since within the observed wavelength regime, the YSOs can be roughly assumed as black-body emitters. The two-component fits with the related temperatures have been added to the SEDs and Table 2. For sources where the data points from different epochs suggest variation between these observations, a second fit has been added to depict the influence 

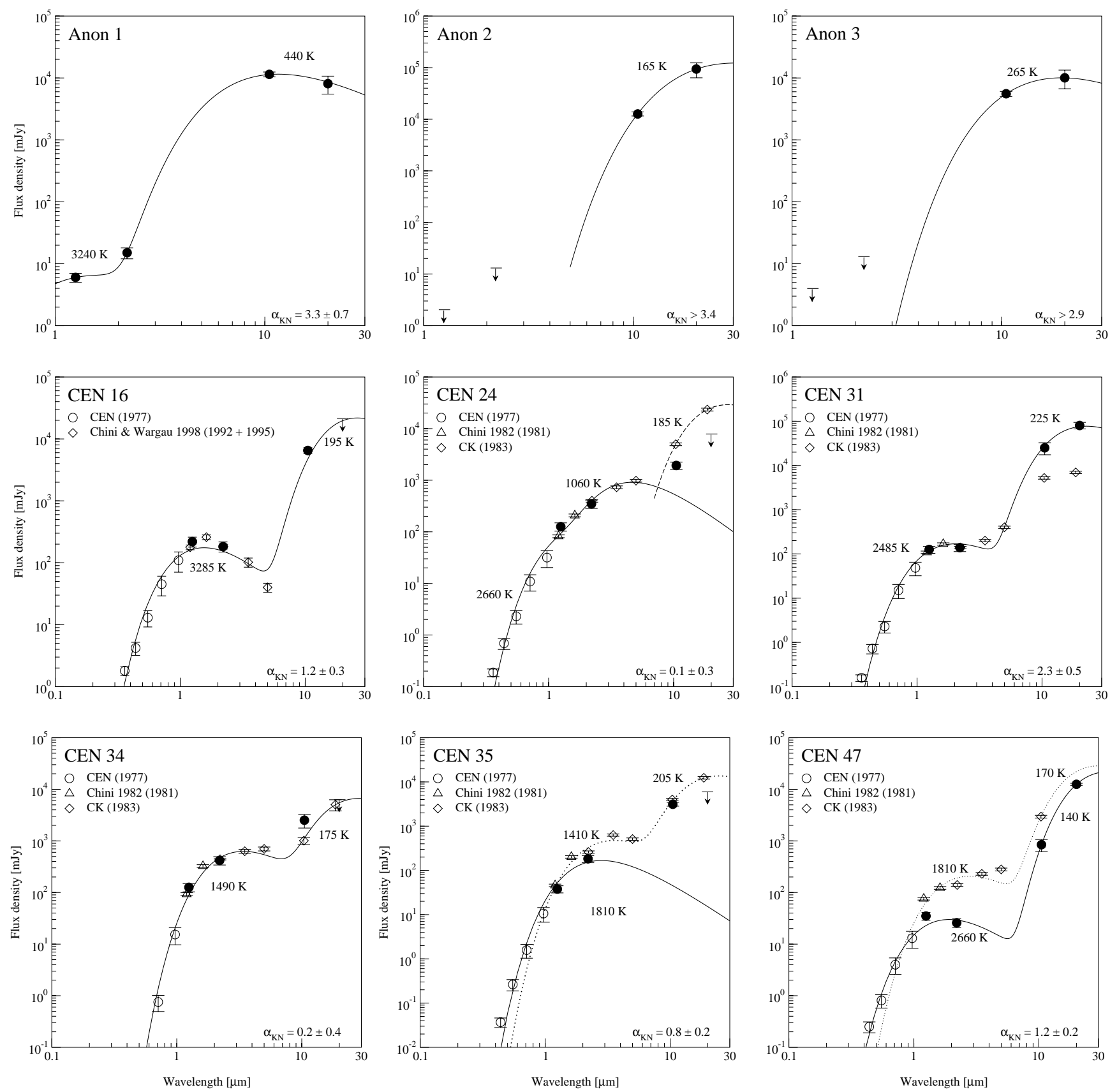

Fig. 3. Spectral energy distributions of the compact sources. Anon 4 has been excluded, as only one well-defined flux value at $N$ could be obtained. The data from the present study are marked with a circle $(\bullet)$. The numbers in brackets within the legends give the epoch of observation. The curves represent the two component black-body fits according to the quoted temperatures.

on the derived parameters (CEN 47, IRS 23, KleinmannWright object). The derived temperatures range from 845 to $3240 \mathrm{~K}$ for the hot component and from 140 to $440 \mathrm{~K}$ for the warm one. The mean values are $1660 \mathrm{~K}$ and $235 \mathrm{~K}$, respectively. Since it is generally assumed that dust grains melt at $\sim 1800 \mathrm{~K}$, the extreme temperatures of $>2500 \mathrm{~K}$ cannot originate from hot dust. Therefore, it is more likely that the circumstellar extinction of the radiation from the stellar surface simulates such high temperatures by diminishing the flux densities at lower wavelengths.

\subsection{Luminosity}

The total luminosity of the YSOs cannot be determined uniquely because of both interstellar and circumstellar dust. Likewise, the distance to M 17 is still under debate. We will address this last issue in Sect. 5 separately and use for the moment a distance of $1.6 \mathrm{kpc}$.

It is very likely that most of the luminosity of the Class I sources is emitted longward of $20 \mu \mathrm{m}$, where observational data do not exist. The results on the cocoon stars, demonstrated by CK, can be used to achieve an 

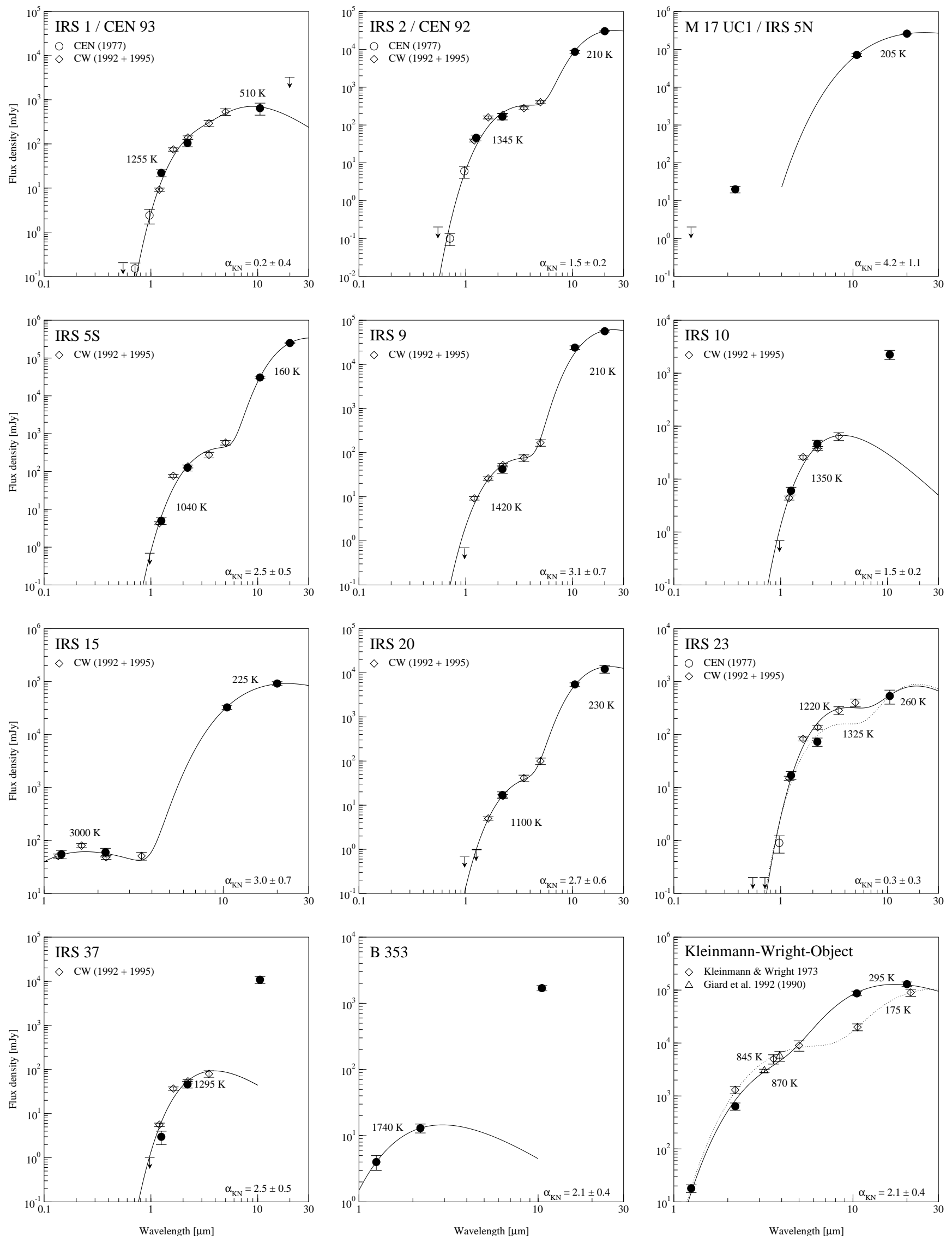

Fig. 3. continued. 
Table 2. Properties of YSOs in M 17 derived from the SEDs. $L_{\mathrm{obs}}$ is the luminosity based on published data and our own measurements taken from the SEDs. The upper limits for the luminosities can be mainly explained by lacking data at shorter wavelengths. Nevertheless, the luminosities can be considered good estimates. The morphology describes the major and minor axis in $\mathrm{AU}(F W H M)$ of the dust emission at $10.5 \mu \mathrm{m}$ (adopting a distance of $1.6 \mathrm{kpc}$ ). Note that an accuracy of $0^{\prime \prime} 1$ is equivalent to 160 AU. Due to diffraction limits, every extent below 1920 AU would result in showing up as a pointsource.

\begin{tabular}{|c|c|c|c|c|c|c|}
\hline \multirow{2}{*}{ Source } & \multicolumn{2}{|c|}{ Spectral Index } & \multicolumn{2}{|c|}{ Temperature } & \multirow{2}{*}{$\begin{array}{l}L_{\mathrm{obs}} \\
{\left[L_{\odot}\right]}\end{array}$} & \multirow{2}{*}{$\begin{array}{c}\text { Morphology } \\
D_{\text {maj }} \times D_{\text {min }}[\mathrm{AU}]\end{array}$} \\
\hline & $\alpha_{K, N}$ & $\alpha_{K, Q}$ & $\begin{array}{l}\text { hot } \\
\text { component }\end{array}$ & $\begin{array}{l}\text { warm } \\
\text { component }\end{array}$ & & \\
\hline KW & $2.1 \pm 0.4$ & $1.4 \pm 0.1$ & 870 & 295 & 2585 & pointsource \\
\hline B353 & $2.1 \pm 0.4$ & - & 1740 & - & 75 & pointsource \\
\hline CEN 34 & $0.2 \pm 0.4$ & $<0.2$ & 1400 & 175 & $<130$ & pointsource \\
\hline CEN 35 & $0.8 \pm 0.2$ & $<0.6$ & 1810 & 205 & $<140$ & pointsource \\
\hline IRS 1 & $0.2 \pm 0.4$ & $<0.6$ & 1255 & 510 & $<60$ & pointsource \\
\hline IRS 2 & $1.5 \pm 0.2$ & $1.4 \pm 0.1$ & 1345 & 210 & 345 & pointsource + filament \\
\hline CEN 16 & $1.3 \pm 0.3$ & $<1.2$ & 3285 & 195 & $<275$ & $8320 \times 6720$ \\
\hline CEN 31 & $2.3 \pm 0.5$ & $3.1 \pm 0.7$ & 2485 & 225 & 910 & $12000 \times 7740$ \\
\hline CEN 24 & $0.1 \pm 0.3$ & $<0.4$ & 2660 & 185 & $<150$ & pointsource \\
\hline CEN 47 & $1.2 \pm 0.2$ & $1.8 \pm 0.2$ & 2660 & 140 & 110 & confused pointsource \\
\hline IRS 5S & $2.5 \pm 0.5$ & $2.4 \pm 0.2$ & 1040 & 160 & 1900 & $4480 \times 4000$ \\
\hline IRS $5 \mathrm{~N}$ & $4.2 \pm 1.1$ & $3.3 \pm 0.4$ & - & 205 & 4775 & $3030 \times 2560$ \\
\hline IRS 9 & $3.1 \pm 0.7$ & $2.3 \pm 0.2$ & 1420 & 210 & 735 & $23040 \times 8480$ \\
\hline IRS 10 & $1.5 \pm 0.2$ & - & 1350 & - & 55 & $3950 \times 2450$ \\
\hline IRS 15 & $3.0 \pm 0.7$ & $2.3 \pm 0.2$ & 3000 & 225 & 1405 & $\begin{array}{l}\text { core: } 5500 \times 5450 \\
\text { envelope: } 8300 \times 6420\end{array}$ \\
\hline IRS 20 & $2.7 \pm 0.6$ & $2.0 \pm 0.2$ & 1100 & 230 & $<165$ & $12340 \times 8820$ \\
\hline IRS 23 & $0.3 \pm 0.3$ & - & 1220 & 260 & $<35$ & pointsource \\
\hline IRS 37 & $2.5 \pm 0.5$ & - & 1295 & - & $<250$ & $17900 \times 11940$ \\
\hline Anon 1 & $3.3 \pm 0.7$ & $1.9 \pm 0.2$ & 3240 & 440 & 590 & $8780 \times 4940$ \\
\hline Anon 2 & $>3.4$ & $>3.0$ & - & 165 & $<1100$ & $\begin{array}{c}\text { core: } 5620 \times 4450 \\
\text { envelope: } 14700 \times 12980\end{array}$ \\
\hline Anon 3 & $>2.9$ & $>2.0$ & - & 265 & $<320$ & filament \\
\hline Anon 4 & $>3.1^{*}$ & - & - & - & $<830$ & $8960 \times 6720$ \\
\hline
\end{tabular}

${ }^{*}$ Spectral index $\alpha_{J N}$.

estimate of the bolometric luminosity of the investigated sources. Such a cocoon star can be regarded as a YSO, surrounded by layers of dust which are confined by evaporation temperatures attained for the different dust components at significant radii from the central star. CK argue that cocoon stars only emit about $10 \%$ of their total energy below $20 \mu \mathrm{m}$. Since representatives of this object class are contained in the present sample, they can be used as templates and their ratio of $L_{\mathrm{obs}} / L_{\mathrm{bol}}$ may serve as an estimate for the remaining YSOs in M 17.

The observed luminosities, as listed in Table 2, range from 55 to $4775 L_{\odot}$ suggesting that the bolometric luminosities are a factor of ten higher. Obviously, the YSOs represent a very young generation of medium to high-mass stars in M 17 that are still enshrouded by the remnants of their protostellar cloud. The fact that some of these objects are seen even at wavelengths below $2.2 \mu \mathrm{m}$ is a result of the ionising radiation and the stellar winds from the nearby O-type stars which have partly cleared the region. We suggest, that these M 17 objects are high mass counterparts of classical Class I sources.

\subsection{Morphology}

The spatial resolution of our MIR images is limited by diffraction and yields values of $1^{\prime \prime} .2$ in $N$ and $2^{\prime \prime} .3$ in $Q$. If we deconvolve the observed profiles of the compact sources with the point source pattern, we find that most of the sources are unresolved. However, there are 6 objects whose 10.5 and $20 \mu \mathrm{m}$ emission is clearly asymmetric with axis ratios of up to $\approx 1.5$. Checking these cases on our $K$-band images, we do not find any double sources. One cannot completely rule out the existence of unresolved multiple MIR sources, where some of the components do not have NIR counterparts. Nevertheless, it is very likely that we are dealing with flattened dust cocoons and/or circumstellar disks. Some of these sources are described in Sect. 4.5.

\subsection{Evolutionary stage}

In order to investigate the evolutionary stage of the infrared sources in M 17, spectral indices $\alpha_{K N}$ and $\alpha_{K Q}$ (see Table 2) and the near-infrared excess ratios according to 
Eq. (2) have been calculated. Within the observational errors, all sources have positive spectral indices. Their visual extinction can be estimated from the TCDs by CW and ranges between $10<A_{V}<40 \mathrm{mag}$, and therefore, the positive spectral index must be largely originating from circumstellar emission and cannot be simulated by heavy reddening. This qualifies all sources to be Class I YSOs. Only CEN 24, CEN 34, IRS 1 and IRS 23 are also consistent with $\alpha_{K N}=0$; the values for $\alpha_{K Q}$ are only upper limits close to zero because there are no detections at $20.0 \mu \mathrm{m}$. Furthermore, these 4 sources appear point-like. This indicates that they are possibly the most evolved stars in the sample and in a transitional stage between Class I and II.

Sources with an optical counterpart have an average $\alpha_{K N}=1.0 \pm 0.8$ and correspond to the cocoon stars. Those sources with only near-infrared counterparts have an average spectral index of $\alpha_{K N}=2.6 \pm 0.9$ and have to be designated as even younger Class I objects. The new sources, Anon 2-3, can only be characterised by the lower limits of their spectral indices, because near-infrared counterparts are still missing. However, in either case they are significantly larger than 2. In summary, the MIR data provide strong evidence that we have discovered an extremely young cluster of Class I sources.

\subsection{Individual YSOs}

In the following section we discuss individual sources of particular interest in more detail. Their choice was either guided by their particular nature or their character as a representative of an object class among the YSOs in M 17.

\subsubsection{17-UC1 and IRS 5S}

The results until the epoch 1998 of the ultra-compact H II region M 17-UC1, or IRS $5 \mathrm{~N}$ and IRS $5 \mathrm{~S}$ in our nomenclature, has been published by Chini et al. (2000). However, the findings from our recent TIMMI2 observations indicate that the $N$ flux of M 17 -UC1 increased by $10 \%$ from 1997 to 2001. The development is demonstrated in Fig. 4.

\subsubsection{Kleinmann-Wright-object}

The Kleinmann-Wright object has been discovered by Kleinmann \& Wright (1973) as one of the brightest infrared sources known so far. It has been classified as a Herbig Be star of spectral type B2. Many efforts have been undertaken to derive its mass loss. The latest attempt has been made by Jinliang et al. (1997). They have combined their own newly developed nonLTE (local thermodynamical equilibrium) model and compared the predictions with observational results quoted in the literature. The preferred quantities were the ratios of several hydrogen lines $(\operatorname{Br} \alpha, \operatorname{Br} \gamma, \operatorname{Pf} \gamma$, $\mathrm{Pa} \delta)$. Finally, they derived a mass-loss rate of $1.3 \times$ $10^{-6} M_{\odot} \mathrm{yr}^{-1}$ which is significantly less than the value

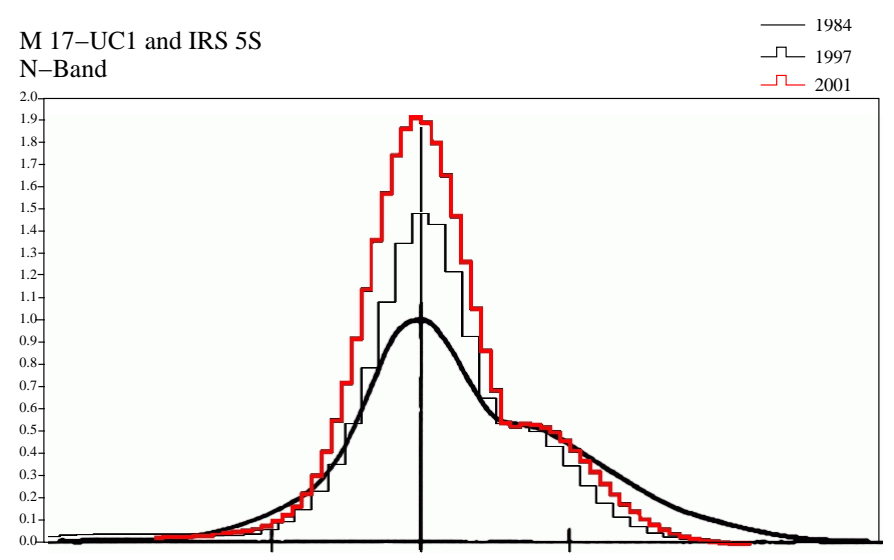

Fig. 4. Overlays of the slit scans by FS (bold curve, flipped in east-west direction) with cuts through the $N$-band maps (histograms) of MANIAC (middle thin line) and TIMMI2 (upper bold line). See also Fig. 4 in Chini et al. (2000).

of $2.2 \times 10^{-6} M_{\odot} \mathrm{yr}^{-1}$ found by Persson et al. (1984). It seems that this decrease over the years has been synchronous with the raise in dust temperature which is obvious from Fig. 3. The exhausting source of extincting circumstellar matter probably cannot compensate the destruction of the dust from the highly energetic stellar radiation. The thermal energy has to be distributed between less particles than before which leads to the dust heating.

\subsubsection{IRS 9}

Figure 5 shows an unpublished $N$-band image of IRS 9, including the nearby cocoon star CEN 31, obtained with TIMMI at the $3.6 \mathrm{~m}$ telescope on La Silla in 1995. The shape of IRS 9 is very eye-catching and is similar to a spiral or hysteresis curve. Unfortunately, the telescope beam was off-centred and so the complete morphology remains unclear. It must be noted that the source is clearly seen in the positive beam and yet it is less obvious in the negative beam. As a result the restoration procedure to a singlebeam map reproduces this spiral well. The MANIAC maps suffer too much from atmospherical instabilities to obtain a proper morphology determination. Instead only flux measurements could be extracted. Further observations are required to verify the shape of the source.

A two-dimensional Gaussian with a FWHM of 14 ". $4 \times 5^{\prime \prime} .3$ could be fitted to IRS 9 in Fig. 5 which is equal to a linear extent of $23040 \times 8480$ AU for the adopted distance of $1.6 \mathrm{kpc}$. Interestingly, recent theoretical studies seem to prove the possibility of building spirals during the formation of binary stars from a common cloudlet. As one example, the numerical simulations of Durisen et al. (2000) demonstrate that starting from a barlike density distribution, rotation and collapse may be responsible for spiral-shaped cloud fragments.

Tsuribe \& Inutsuka (2000) investigated the evolution of protostellar cloud fragments with respect to binary star formation by semi-analytic numerical simulations. By considering about 100 initial conditions, they were able to 


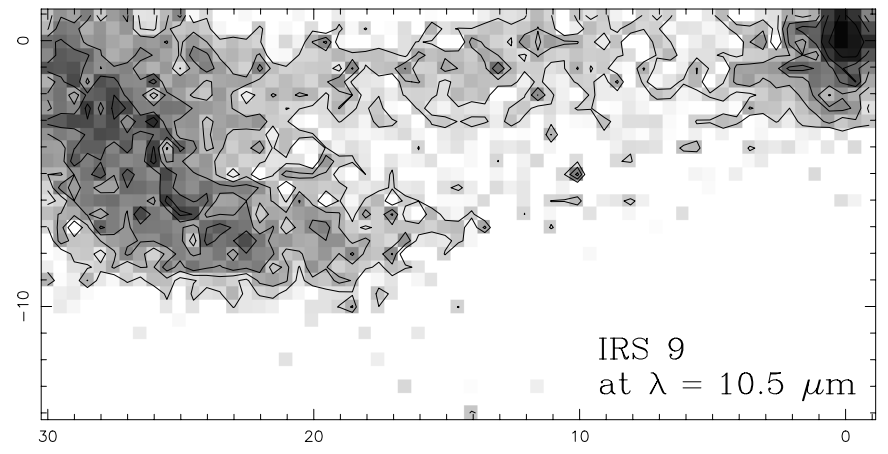

Fig. 5. $N$ band image of IRS 9 (left) and CEN 31 (right), taken with TIMMI in 1995. The structure similar to a hysteresis curve is regarded as a possible mode during early the stages of binary star formation, as predicted from modern models. The lowest contours represent the $3 \sigma$ detection limit.

create a systematical catalogue of density distributions. Again, spiral structures appeared. Using this argument, the shape of IRS 9 might be consistent with a collapse during relatively strong rotation, whereby a single adiabatic core could be built.

However, whether binary stars can evolve from protostellar spiral-shaped clouds or not, is not of primary interest for IRS 9. Rather it has been demonstrated that dust morphologies shaped as spirals are possible and it seems that IRS 9 might be the first observed example for this.

\subsubsection{IRS 15}

The work of CK has clearly answered the long lasting question: Are high-mass stars capable of hosting larger amounts of circumstellar dust? Not only can the remnants of the protostellar collapse survive the strong stellar radiation from the typically early B-type stars for a significant amount of time, they also tend to build several layers ( $\mathrm{co}$ coons), confined by the melting temperatures of the dust components. The modelled radii ranged from some tens of $\mathrm{AU}$ for the inner cocoons to a few thousands of $\mathrm{AU}$ for the outer ones. Unfortunately, the spatial resolution is too low to verify most of them directly from imaging.

Taking rotation of the circumstellar envelopes into account, the detection of circumstellar disks does not seem to be such a big surprise. However, only a handful of examples are known for massive stars today. This implies that it is either a very rare phenomenon or the lifetime of such a disk is very short. Nevertheless, a possible candidate for a circumstellar disk is IRS 15, as shown in Fig. 6. Here, the left image displays a compact central source surrounded by an extended envelope. In case of a disk, the projected linear extents would be $8300 \times 6420 \mathrm{AU}(F W H M)$. After subtracting the central spherical core with a diameter of $5500 \mathrm{AU}(F W H M)$, the envelope visible in the right image remains. By assuming a circular disk, the axis ratio of 1.3 leads to an inclination angle of about $40^{\circ}$, defined as the angle to the plane of the sky. The spectral index in Table 2 clearly classfies IRS 15 as an extreme Class I

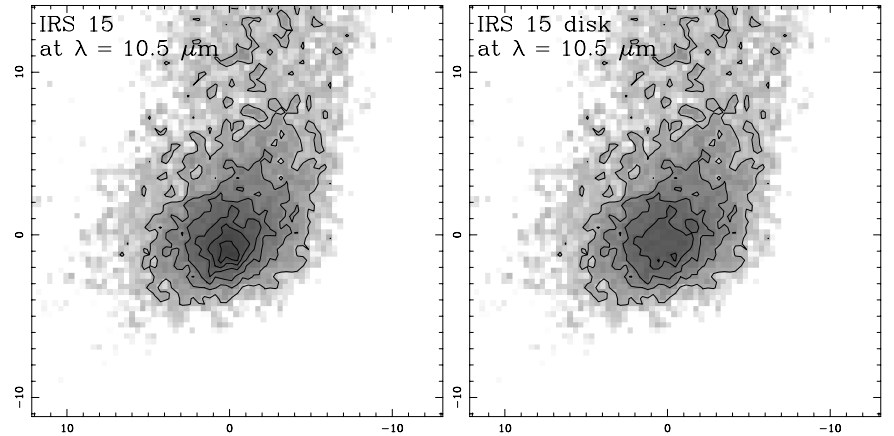

Fig. 6. $N$ band images of IRS 15 . After subtracting the pointlike compact core from the original image (left), a large flattened envelope remains (right) which obviously dominates the mid-infrared emission.

source. And with a flux density of $7185 \pm 790 \mathrm{mJy}$, even the spherical core alone must be regarded as a Class I YSO with a resulting spectral index of $\alpha_{K N}=2.1 \pm 0.4$.

\section{The distance to $\mathrm{M} 17$}

CEN derived a distance of $2.2 \pm 0.2 \mathrm{kpc}$ based on $U B V$ photometry of 19 possible early type stars. Hanson et al. (1997) (hereafter HHC) derived a distance of $1.3_{-0.2}^{+0.4} \mathrm{kpc}$ based on published apparent $K$ magnitudes and absolute $K$ magnitudes derived from $K$-band spectroscopy. HHC criticised the distance estimate and the spectral classification of CEN by making three wrong statements. We presume that they originate from several misunderstandings, which must be clarified:

1. HHC argue that a photometric distance determination which relies on both dereddening and estimating spectral types is generally a questionable method. This is wrong - as long as the $U B V$ photometry refers to spectral types earlier than B3 (which allows for a unique solution) the accuracy of the results is comparable to MK classification;

2. HHC state that CEN included stars with only VRI colours in their distance estimate for the cluster. This is not the case, because CEN clearly explained that they used only stars with $U B V$ data (see their Table 3 and their Fig. 6);

3. HHC point out that CEN incorrectly identified numerous cool giant stars for heavily reddened $\mathrm{O}$ stars. Yet in fact two stars CEN 33 (B 324) and CEN 102 (B 305) whose SEDs are compatible with B2 and O5, respectively, turned out to be cool giants by means of the $K$-band spectroscopy. It should be noted that CEN derived those spectral types in the absence of $U B V$ data and by assuming that they are cluster members of luminosity class V. A third star under debate is CEN 92 (B 331) whose SED is compatible with B0. $\mathrm{HHC}$ did not derive a spectral type but agree that the $K$-band spectrum is consistent with a young stellar object with a SED of late O type. 
In order to obtain a proper distance estimate of M 17 , we re-addressed this problem by using all the information available on the cluster members to date. CEN obtained the cluster distance from 19 possible early type stars with $U B V$ photometry and with a reddening that exceeded the foreground extinction of $A_{V} \geq 3.1 \mathrm{mag}$ towards M 17. In this context, the visual extinction and thus the reddening law plays an important role. In order to account for the abnormal reddening law for the dust within $\mathrm{M} 17$, they divided the observed reddening into two parts: i) $E_{B-V} \leq 1$ that originates from the diffuse dust in the foreground, characterised by $R=3.1$, ii) $E_{B-V}>1$ that is due to abnormal dust within M 17 , characterised by $R=4.2$; the latter value was based on $R$ and $I$ data only.

Meanwhile, CW have improved the investigation of the extinction law in M 17 and extended the observed wavelength range until $4.8 \mu \mathrm{m}$. This led to an $R$-value of 4.8 which increases the visual extinction of those stars that are heavily embedded in the abnormal dust and thus reduces their distance correspondingly. By re-calculating the total visual extinction for those CEN stars with unique spectral type solutions from $U B V$ photometry (their Table 3), we derive the results summarised in Table 3.

In general, the spectral types from the different methods agree. Comparing the distance moduli derived in Table 3 with the results of HHC and Hanson \& Conti (1997), there is also good agreement apart from CEN 25 (B 164); the mismatch of about 1.1 mag originates from the different $A_{V}$ values assigned: while $\mathrm{CW}$ use a common $R$-value of 4.8 with only little scatter across M 17, HHC use individual values for each star. Averaging the distances for those 12 stars from Table 3 without known IR excess we derive a value of $1.6 \pm 0.3 \mathrm{kpc}$ in agreement with the result by HHC. Interestingly, the IR excess objects CEN 28 and 31 have significantly larger distance moduli than the average. This could indicate a contribution of grey extinction which leads to an underestimate of $A_{V}$. It cannot be excluded that CEN 25, 45 and 58 show a similar behaviour although the data of $\mathrm{CW}$ do not indicate any excess emission. In this case, the distance of $1.6 \mathrm{kpc}$ would be only an upper limit.

\section{Conclusions}

Our MIR imaging has shown for the first time a detailed picture of the distribution of warm dust emission in M 17. In addition, we could obtain for the first time high spatialresolution photometry at MIR wavelengths in this region. Our results lead to the following picture:

1. We have imaged an emission ridge of warm dust $(\sim 230 \mathrm{~K})$ with an extent of 4.5 at MIR wavelengths. It is compressed by an ionisation front which is driven by CEN 1.

2. We have investigated 22 compact sources which all are consistent with the Class I criterion; four of them seem to be in a transitional stage from Class I to II. Hence,
Table 3. The distance to $M$ 17. The refrences for the values within the columns are as follows: (1) numbering by CEN, (2) numbering by Bumgardner (1995), (3) UBV photometry (CEN), (4) MK classification (HHC), (5) $K$-band spectroscopy (HHC), (6) CEN, (7) CEN, (8) this paper, (9) HHC, (10) this paper.

\begin{tabular}{|r|r||r|c|c|r|c|r|c|c|}
\hline C & \multicolumn{1}{|c||}{ B } & \multicolumn{2}{|c|}{ Spectral Type } & $m_{V}$ & $E_{B-V}$ & $A_{V}$ & $M_{V}$ & $D M$ \\
$(1)$ & $(2)$ & $(3)$ & $(4)$ & $(5)$ & $(6)$ & $(7)$ & $(8)$ & $(9)$ & $(10)$ \\
\hline 1 & 189 & O4 & O5 & O3-O4 & 14.13 & 2.49 & 10.25 & -5.18 & ${ }^{2} 9.81$ \\
2 & 111 & O5 & O5 & O3-O4 & 11.03 & 1.48 & 5.40 & -4.88 & 10.51 \\
3 & 98 & O8 & O9 & O9-B1 & 9.92 & 1.11 & 3.63 & -3.64 & 9.93 \\
16 & 311 & B0 & & O9-B2 & 13.69 & 1.65 & 6.22 & -2.86 & 10.33 \\
17 & 336 & B3 & & & 14.96 & 1.29 & 4.49 & -0.78 & 11.25 \\
18 & 260 & O9 & O8 & O3-O4 & 14.20 & 1.98 & 7.80 & -3.96 & 10.36 \\
22 & 302 & B5 & & & 14.81 & 1.16 & 3.87 & -0.21 & 11.15 \\
24 & 275 & B2 & & & 15.55 & 1.75 & 6.70 & -1.35 & 10.20 \\
25 & 164 & B3 & O8 & O7-O8 & 15.41 & 1.77 & 6.80 & -3.96 & 12.57 \\
26 & 253 & B3 & & & 15.74 & 1.60 & 5.98 & -0.78 & 10.54 \\
28 & 150 & B0 & & & 15.56 & 1.32 & 4.64 & -2.86 & 13.78 \\
31 & 289 & B5 & O9.5 & & 15.55 & 1.72 & 6.56 & -3.48 & 12.47 \\
44 & & B3 & & & 15.28 & 1.84 & 7.13 & -0.78 & 8.93 \\
45 & & B1 & & & 13.22 & 1.06 & 3.39 & -2.01 & 11.84 \\
48 & 248 & B2 & & & 15.44 & 1.72 & 6.56 & -1.35 & 10.23 \\
58 & & B3 & & & 15.80 & 1.15 & 3.82 & -0.78 & 12.76 \\
\hline
\end{tabular}

* This value was derived by taking into account that CEN 1 is a double star with comparable components.

the stellar population of M 17 appears to comprise some of the youngest massive YSOs detected so far.

3. The formal colour temperatures of the circumstellar environment as derived from Planck models range from $140 \mathrm{~K}$ to $440 \mathrm{~K}$ for the warm component, and from $845 \mathrm{~K}$ to $3240 \mathrm{~K}$ for the hot component. The observed luminosities exhibit values from 55 to $4775 L_{\odot}$.

4. 12 of the 22 sources show extended MIR emission within our spatial resolution of 1 !'2. Six of them even show an elongated emission morphology, suggesting flattened envelopes, disks or unresolved multiple sources.

5. The distance towards M 17 has been determined to be $1.6 \pm 0.3 \mathrm{kpc}$. The decrease compared to the early observations by CEN results from both a better determination of the extinction law $(R=4.2$ vs. $R=4.8)$ and the elimination of IR excess objects (which tend to be dimmer probably due to grey extinction).

Acknowledgements. We want to thank the programme committee of the MPIfA for the generous allocation of observing time at the $2.2 \mathrm{~m}$ telescope on Calar Alto. Special thanks go to Drs. Th. Böker, A. Krabbe, Th. Lehmann and Th. Nikola for their assistance and advice during the observations with MANIAC and the calibration of the data. It is a pleasure for $\mathrm{MN}$ to thank Dr. R. Zylka for providing and adapting his program package MOPSI which enabled us to reduce the MANIAC data in a straightforward manner. We also thank Dr. M. Felli for the inspiring and constructive discussions. RC wants to acknowledge the financial support by the Deutsche Forschungsgemeinschaft which allowed to cover the costs of the 
MANIAC observing run. MN thanks K. Brooks for the final proof-reading of the manuscript.

\section{References}

Böker, T., Storey, J. W. V., Krabbe, A., \& Lehmann, T. 1997, PASP, 109, 827

Bumgardner, T. E. 1992, M.S. Thesis, Ohio State Univ., Columbus

Bunn, J. C., Hoare, M. G., \& Drew, J. E. 1995, MNRAS, 272, 346

Chini, R., Elsässer, H., \& Neckel, Th. 1980, A\&A, 91, 186 $(\mathrm{CEN})$

Chini, R. 1982, A\&A, 110, 332

Chini, R., \& Krügel, E. 1985, A\&A, 164, 175 (CK)

Chini, R., \& Wargau, W. F. 1998, A\&A, 329, 161 (CW)

Chini, R., Nielbock, M., \& Beck, R. 2000, A\&A, 357, L33

Durisen, R. H., Pickett, B. K., Bate, M. R., et al. 2000, Dynamic Instability of Barlike Modes, in Birth and Evolution of Binary Stars, ed. B. Reipurth, \& H. Zinnecker, Post. Proc. IAU Symp., 200, 187

Felli, M., Johnston, K. J., \& Churchwell, E. 1980, ApJ, 242, L157

Felli, M., \& Stanga, R. 1987, A\&A, 175, 193 (FS)

Gehrtz, R. D., Hackwell, J. A., \& Jones, T. W. 1974, ApJ, 191, 675
Hanner, M. S., Tokunaga, A. T., Veeder, G. J., \& A'Hearn, M. F. 1985, AJ, 89, 162

Hanson, M. M., \& Conti, P. S. 1995, ApJ, 448, L45 (HC)

Hanson, M. M., Howarth, I. D., \& Conti, P. S. 1997, ApJ, 489, 698 (HHC)

IRTF Bright Infrared Standard Stars 1990, NASA Infrared Telescope Facility, Mauna Kea, Hawaii, USA, http://irtf.ifa.hawaii.edu/online/IRTF/ Catalogs/bright_standards

Jinliang, H., Dongrong, J., \& Chengqi, F. 1997, A\&A, 327, 725

Kleinmann, D. E., \& Wright, E. L. 1973, ApJ, 185, L131

Lada, C. J. 1987, Star Formation: From OB Associations to Protostars, in Star Forming Regions, ed. M. Peimbert, \& J. Jugaku, IAU Symp., 115, 1

Persson, S. E., Geballe, T. R., McGegor, P. J., Edwards, S., \& Lonsdale, C. J. 1984, ApJ, 286, 289

Rieke, G. H., Lebofsky, M. J., \& Low, F. J. 1985, AJ, 90, 900

Tokunaga, A. T. 1984, AJ, 89, 172

Tsuribe, T., \& Inutsuka, S. 2000, On the Condition of Binary Fragmentation, in Birth and Evolution of Binary Stars, ed. B. Reipurth, \& H. Zinnecker, Post. Proc. IAU Symp., 200, 184

Vallée, J. P., \& Bastien, P. 1996, A\&A, 313, 255

Zylka, R. 1995, Pocket Cookbook for the Mapping Software, Max-Planck-Institut für Radioastronomie, Bonn, Germany 